\section{Förderungspreis 2000 der Croma Pharma GmbH}

Herr Mag. Gerhard Prinz und seine Firma Croma Pharma haben auch heuer wieder den Förderungspreis für eine bedeutende wissenschaftliche Arbeit auf dem Gebiet der Augenheilkunde in der Höhe von ATS 50.000,- gestiftet. Die ÖOG dankt Herrn Mag. Prinz für diese großzügige Stiftung umso mehr, als seine Firma einen privatwirt schaftlichen Familienbetrieb darstellt.

Dieser Preis wurde in unserem Spektrum der Augenheilkunde ausgeschrieben. Acht junge Autoren reichten dafür ihre Arbeiten ein.

Diese Arbeiten wurden einer Jury aus den österreichischen Augenkliniken zur Begutachtung vorgelegt. Die Bewertung ergab schließlich eine gleichwertige Reihung zweier Arbeiten auf dem ersten Platz:

Johannes Nepp: Psychovegetative Spannung bei Keratoconjunctivitis sicca und Beeinflussung mittels Akupunktur.

Wolfgang Radner: Ein neues visuell-akustisch gestütztes Computeranalyseverfahren zur exakten Messung der Lesegeschwindigkeit und zur erleichterten Datenverwaltung bei der Bestimmung des Lesevermögens.

Dr. Johannes Nepp untersuchte in seiner Arbeit die klinische Wirksamkeit der Akupunktur bei Keratoconjunctivitis sicca (= KCS, Syndrom des trockenen Auges - die Binde- und Hornhaut werden durch eine verminderte Tränensekre-tion nur mangelhaft benetzt). Die Ergebnisse weisen erstmals den positiven Einfluss der Akupunktur auf die vegetativen und psychischen Spannungen bei dieser Erkrankung aus.

Dr. Wolfgang Radner entwickelte in nur drei Jahren ein neues, visuell und akustisch gestütztes Computeranalyseverfahren, das viel genauer als andere Verfahren die Bestimmung des Lesevermögens selbst unter ungünstigen Bedingungen wie Lärmüberlagerungen messen kann.

Die Überreichung der Preisgelder an die Autoren durch Herrn Mag. Prinz erfolgte bei der Vollversammlung der ÖOG am Freitag, dem 2. Juni 2000 in der Universität Wien.

Die beiden Arbeiten werden in diesem Heft publiziert.

\section{Förderungspreis 2001 der Croma Pharma GmbH}

Die Croma Pharma setzt einen Förderungspreis für eine bedeutende wissenschaftliche Arbeit auf dem Gebiet der Augenheilkunde in der Höhe von

\section{öS 50.000,00}

aus.

\section{Teilnahmebedingungen:}

1. Die Bewerber müssen das medizinische Hochschulstudium erfolgreich abgeschlossen haben, die Österreichische Staatsbürgerschaft besitzen und dürfen zum Zeitpunkt des Stichtages (siehe Punkt 2.) das 45. Lebensjahr noch nicht vollendet haben; sie dürfen weder Abteilungsleiter noch Klinikchef sein.

2. Die eingereichte Arbeit muss in Maschinschrift hergestellt sein; sie darf jedoch bereits innerhalb der letzten 2 Jahre publiziert worden sein. Die Arbeit muss bis spätestens

\section{März 2001}

bei der Österreichischen Ophthalmologischen Gesellschaft, Schlösselgasse 9, 1080 Wien, unter Hinweis auf die Bewerbung zum „Croma Pharma-Förderungspreis“ in einem verschlossenen Umschlag einlangen. Die Arbeit ist ein kurzer Lebenslauf des Verfassers beizulegen. Die Einrichtung wird vertraulich behandelt.

3. Über die Zuerkennung des Preises entscheidet eine Jury der Österreichischen Ophthalmologischen Gesellschaft. Bei gleichwertigen Arbeiten ist der praxisbezogenen der Vorzug zu geben.

4. Die Jury ist berechtigt, den Preis auf mehrere Verfasser zu verteilen; sie kann aber auch von der Zuerkennung des Preises absehen, wenn sie zur Überzeugung gelangt, dass keine preiswürdige Arbeit vorliegt.

5. Die Jury entscheidet mit einfacher Stimmenmehrheit. Ihre Entscheidung ist endgültig und unterliegt keinerlei Anfechtung, insbesondere auch nicht vor Gericht.

6. Die Croma Pharma ist berechtigt, den Inhalt der Arbeiten von Preisträgern im Rahmen der Aktivitäten der Croma Pharma zu verwerten, diese ganz - oder teilweise - zu veröffentlichen und Preisträgern einzuladen, über das Thema ihrer Arbeit einen Vortrag zu halten. 